Published in Phys. Rev. B 76, 205437 (2007)

\title{
Quantum size effects in solitary wires of bismuth
}

\author{
Shadyar Farhangfar $*$ \\ Department of Physics, University of Jyväskylä, FIN-40014 Jyväskylä, Finland and \\ Max Planck Institute of Microstructure Physics, Weinberg 2, D-06120 Halle, Germany
}

(Dated: November 16, 2018)

\begin{abstract}
We have performed four-probe electrical transport measurements on solitary highly crystalline wires of semimetallic bismuth with aspect ratios up to 60 at room and at cryogenic temperatures. By proper choice of the substrate material and the film deposition parameters, lithographic wires with lateral dimensions of down to one single grain, $\sim 250 \mathrm{~nm}$, were fabricated. The electrical resistance of each wire was measured against its thickness through successive reactive ion etching of the self-same wire. Quantum size effects revealed themselves as regular oscillations in the electrical resistance. Some evidence for the semimetal-to-semiconductor phase transition has been detected. The measured data are discussed within the framework of the existing theoretical models.
\end{abstract}

PACS numbers: 73.63.Nm, 73.23.-b, 73.43.Nq

Bismuth is a semimetal with unique magnetic, electrical, and thermal properties. As prime examples, one can mention the large mean free path, large carrier mobility, small effective mass components of the charge carriers, and large magnetoresistance. These unusual properties arise from the highly anisotropic Fermi surface of this material. The small effective mass leads to a large de Broglie wavelength, $\sim 40 \mathrm{~nm}$, making this element a potential choice for the experimental study of quantum confinement effects. Furthermore, the exceptionally low magnetic susceptibility of bismuth and its very low thermal conductivity favor its use in the investigation of certain other phenomena. While the former can be exploited for the study of magnetic impurity effects, the latter is, in turn, of vital importance in thermoelectric applications.

In a microscopic semimetal, as the size of the sample shrinks, the overlaps between the valence and the conduction bands become smaller, evolving ultimately to the formation of a semiconductor energy gap. Further reduction of the band overlaps will lead to a semiconductorto-insulator phase transition [1, 2]. In principle, a whole quantum circuit, consisting of (semi)metal, semiconductor, and insulator components, can be made just by tailoring the energy bands in a bismuthlike semimetal.

Experimental studies of the quantum size effects (QSEs) in two-dimensional (2D) films of bismuth can be traced back to the mid 1960s 1], followed by a theoretical model to describe the measured data soon after 2]. Since then, numerous investigations have been performed to discover transport properties of bismuth at low dimensions. While study of the 2D films has been a subject of steady interest [1]-[5], the one-dimensional (1D) structures have been tackled only recently [6]-[13]. Yet, despite all the advances in microfabrication techniques, experimental evidence for the observation of the QSEs in the $1 \mathrm{D}$ regime has been scarce. This is mostly due to the lack of satisfaction of at least one of the following neces- sary conditions: the lateral dimensions of the sample $w$ and $t$ have to be comparable in size to the carrier wavelength $\lambda$; the energy level spacing $\delta E$ has to be larger than the thermal broadening $k_{\mathrm{B}} T$, and it has to meet the uncertainty principle, $\delta E \gtrsim \hbar / \tau$ ( $\tau$ is the relaxation time); the surface roughness of the wire $\eta$ is to be smaller than $\lambda$; and the fabricated wires should have comparable band structures and morphologies. In addition, the wires have to be contacted in such a way as to avoid screening effects arising from the formation of oxide or Schottky barriers. In what follows, we will first discuss the accomplishment of these conditions in our experiments. We will then introduce our measured oscillations of the electrical resistance as a manifestation of the quantum size effects. Throughout the paper, theoretical arguments will be employed to elucidate the experimental data.

The samples were fabricated by electron beam lithography and thermal evaporation techniques. To obtain wires with the desired quality, i.e. wires with large grain size, low carrier concentration, and small surface roughness, we examined the suitability of different substrates and deposition parameters. Figure 1 illustrates the dependence of the carrier concentration $n$ and the electrical resistivity $\rho$ on the substrate material and its temperature. The carrier densities were obtained from Hall measurements performed at liquid helium temperature on macroscopic samples grown under similar conditions. The extracted resistivities have to be compared to the values obtained for high-quality polycrystalline, $0.5 \times 10^{-5} \Omega \mathrm{m}$, and single-crystalline, $4 \times 10^{-5} \Omega \mathrm{m}$, films 4]. Wires with the largest grain sizes (and lowest carrier concentration) were obtained through the evaporation of bismuth at a rate smaller than $1 \AA^{-1}$ and at a vacuum pressure of better than $10^{-6}$ mbar onto a mica substrate kept at $90^{\circ} \mathrm{C}$. The films grown on mica substrate were n-type, i.e. electrons were the majority charge carriers. The optimal deposition temperature is in accordance with the recommended range for the growth of crystalline 


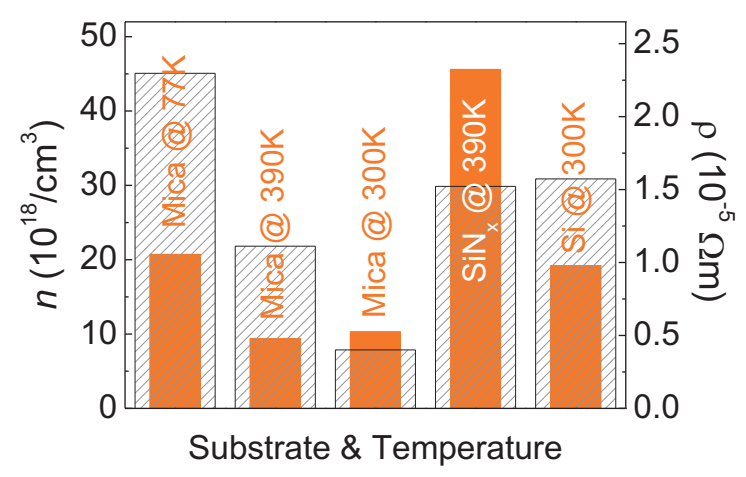

FIG. 1: (Color online) Dependence of the carrier density $n$ (solid histograms) and the resistivity $\rho$ (dashed histograms) on the substrate material (mica, silicon, or silicon nitride) and its temperature.

films, $\frac{1}{3} T_{\mathrm{m}}<T_{\mathrm{S}}<\frac{2}{3} T_{\mathrm{m}}$, where $T_{\mathrm{s}}$ is the substrate temperature and $T_{\mathrm{m}}$ is the melting temperature of the material to be deposited [14]. Prior to the film deposition, to provide a cleaner interface for the growth, the resist and developer remnants in the exposed areas were removed in an oxygen plasma ambiance. Figure 2 shows an atomic force microscope (AFM) image of the wire segment so obtained. The constituent grains have a typical feature size of about $200-300 \mathrm{~nm}$. Initially, prior to the first electrical characterizations, the samples were further analyzed under AFM. While the asperity is of the order of $10 \mathrm{~nm}$ (cf. the carrier wavelength) for the films deposited onto a mica substrate in the aforementioned conditions, it can often be much larger for those grown onto the other substrates and/or in different conditions. Displayed in Fig. 3 is the x-ray diffraction (XRD) pattern of a bismuth film grown onto a mica substrate. Clearly, the films are highly crystalline and have a preferred growth orientation. It would also be desirable to have transmission electron microscope (TEM) images or scattering patterns of the films as well; however preparation of the samples grown on mica for TEM characterization proved problematic [15]. It is also worth mentioning, that despite all our efforts (all together more than 100 samples went through the metalization procedure), the narrowest wires were more than $200 \mathrm{~nm}$ wide [full width at half maximum, (FWHM)]. The main obstacle here was charging of the mica substrate during the e-beam lithography process, which in turn led to the backscattering of the electron beam and, consequently, broadening of the exposed areas. Nevertheless, that small fraction of the wires with narrower widths subsequently failed to survive through the lift-off process or the following etching steps. However, as shown in Fig. 4, the calculated density of states

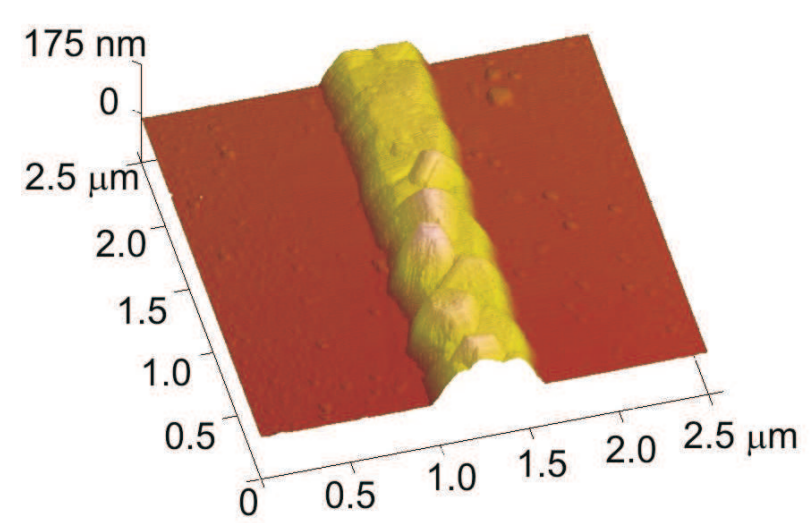

FIG. 2: (Color online) Atomic force microscope image of a bismuth wire segment deposited onto a mica substrate. The substrate temperature was $90^{\circ} \mathrm{C}$. The grains have a feature size larger than $200 \mathrm{~nm}$. The wires have trapezoidal cross sections with sharply rising sidewalls.

$D(E)$ for a 1D bismuth wire with dimensions comparable to those in the present study suggests feasibility of the detection of QSEs in wires as wide as $300 \mathrm{~nm}$. The level spacings here are in the range $1-10 \mathrm{meV}$, being an order of magnitude larger than the thermal broadening, $k_{\mathrm{B}} T \sim 0.4 \mathrm{meV}$, at liquid helium temperature.

Formation of oxide or Schottky barriers between the wires and the contact leads was circumvented by reducing the film deposition procedure to a single step. In fact, observation of the low-bias plateaus in the currentvoltage $(I V)$ characteristics of the recent experiments [9, 11] may be attributed to the existence of such barriers. Figure 5 depicts the $I V$-characteristic of a sample measured in liquid helium. The curve is linear down to the lowest bias currents. Subsequent to the film deposition and the lift-off, each sample was measured at room, at liquid nitrogen, and at liquid helium temperatures. All the measurements were performed in four-probe configuration, most of them through both the ac and the dc techniques. To contact the samples to the measuring setup, we used conductive carbon paste. This material makes a robust contact to the pads and has a negligible contact resistance. Due attention was paid not to heat the samples by large currents; typical injection currents were in the range of $1-20 \mathrm{nA}$. The reference frequencies in the ac-measurements were $10-20 \mathrm{~Hz}$. After each series of measurements at the specified temperatures, the sample was made thinner in a reactive ion etching machine. To reduce the sample thickness by about $5-8 \mathrm{~nm}$, etching was done for a duration of $30 \mathrm{~s}$ with a $50 \mathrm{~mW}$ of argon plasma power. The temperature during this process was $30^{\circ} \mathrm{C}$. Our samples were typically $100-150 \mathrm{~nm}$ thick, $\sim 300 \mathrm{~nm}$ wide (FWHM), and 10-20 $\mu \mathrm{m}$ long. The electrical resistances of such wires were in the range 


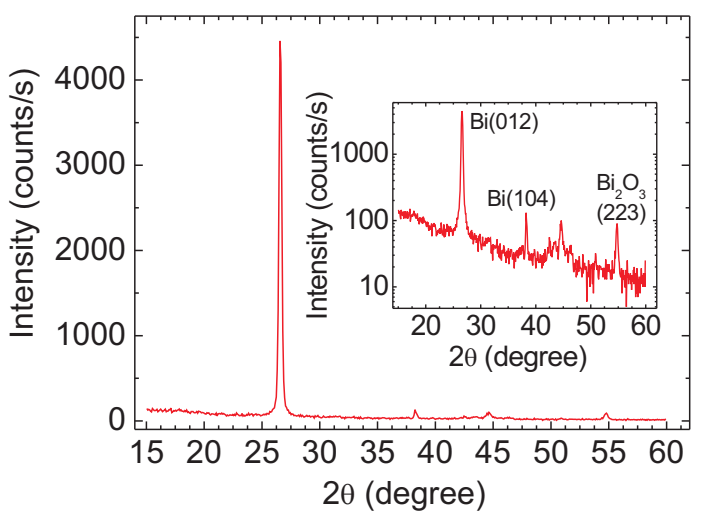

FIG. 3: (Color online) X-ray diffraction pattern of a bismuth film grown on a mica substrate. The film has a well-defined growth orientation (012). To make the smaller peaks visible, the vertical axis in the inset is presented on a logarithmic scale. The Miller indices corresponding to the different orientations are taken from the JCPDS data base (codes 05-0519 for the $\mathrm{Bi}$ and 29-0236 for $\mathrm{Bi}_{2} \mathrm{O}_{3}$ ). The third peak from the left was not identified.

$1-10 \mathrm{k} \Omega$ at room temperature. Most of the wires exhibited a resistance increase of up to even $100 \%$ at $T \approx 77$ $\mathrm{K}$ followed by a further increase of about $10-30 \%$ at $T \approx 4.2 \mathrm{~K}$. In contrast to this semiconductorlike behavior, occassionally there were also samples reminicent of the conventional metals. In what follows, we will consider samples of the former type.

Starting with an initial thickness of $100-150 \mathrm{~nm}$, a series of typically $8-15$ measurements were performed until the sample resistance grew exponentially. (Indeed, in most cases the samples were broken prior to this abrupt resistance increase.) Before proceeding to a more detailed discussion of the measured data in the following, it would be illuminating to exclude the possibility that the observed oscillations could have stemmed from stochastic variations in the cross sections of the wires and the corresponding fluctuations in the electrical resistances. The resistance of the wire at the $i$ th etching step can be written as $R_{i} \approx \rho_{i} l /\left[w\left(t_{i} \pm \eta_{i}\right)\right]$. Here, $l, w$, and $t_{i}$ stand for the length, width, and the thickness of wire respectively; $\eta_{i}$ is the asperity, and $\rho_{i}$ is the corresponding electrical resistivity. The alteration in the resistivity of wire as its surface roughness changes can be estimated through the expression $\rho_{i}^{-1} \sim \rho_{0}^{-1}\left[1-\frac{3}{16}\left(1-p_{i}\right) \frac{\Lambda_{0} P_{i}}{S_{i}}\right]$, where $P_{i}$ is the perimeter, $S_{i}$ is the cross-section, and $p_{i}$ is the surface polish (a measure of the surface smoothness at the $i$ th etching step; $p_{i}=1$ for the specular reflection of the carriers from the surface). Furthermore, $\rho_{0}$ refers to the resistivity of a wire with perfect surface reflectivity and $\Lambda_{0}$ is the corresponding carrier mean free

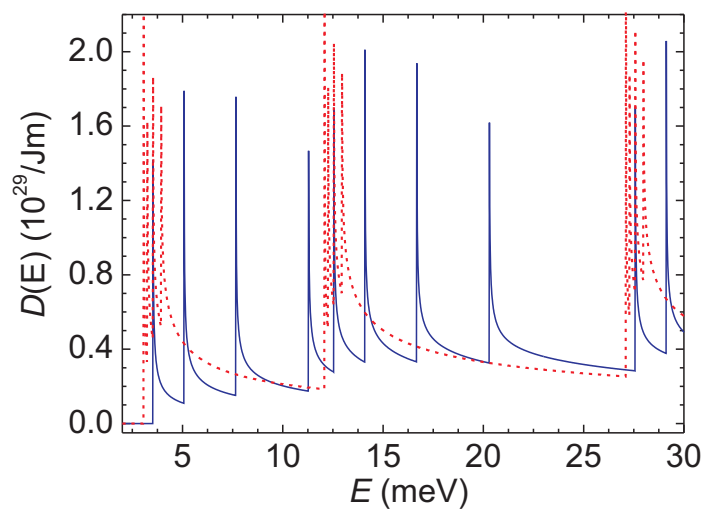

FIG. 4: (Color online) Calculated density of states $D(E)$ for a one-dimensional $(l \gg w, t)$ bismuth wire with lateral dimensions $w=300 \mathrm{~nm}, t=50 \mathrm{~nm}$ (solid blue line), and $t=150$ $\mathrm{nm}$ (dashed red line). The wire was aligned along the bisectrix axis. The values for effective masses $m_{x}=0.00139$, $m_{y}=0.291$, and $m_{z}=0.0071$ (in units of the free electron mass) are taken from Ref. [16]. For clarity, only the contribution of the few first subbands is shown.

path 17]. Accordingly, within the course of two successive etching steps, the resistivity of wire remains almost intact, $\rho_{i+1} \approx \rho_{i}$. In particular, in the beginning of the etching process, for a relatively smooth wire surface, one has $\rho_{i} \sim \rho_{0}$. It is now straightforward to show that the necessary condition for the electrical resistance to drop in a subsequent step, i.e. to have $R_{i+1} \lesssim R_{i}$, is that the average thickness removal between the two steps $\left|\Delta t_{i}\right|$ is smaller than the difference between their corresponding asperities, $\left|\Delta t_{i}\right| \lesssim \pm\left(\eta_{i+1}-\eta_{i}\right)$, a condition that can hardly be satisfied. Nonetheless, the stochastic variations in the wire cross section can barely reveal themselves as regular oscillations with a clear periodicity in the thickness.

Figure 6(a) shows measured oscillations of the electrical resistance as a function of the wire thickness. The sample was originally $14 \mu \mathrm{m}$ long, $320 \mathrm{~nm}$ wide, and 100 $\mathrm{nm}$ thick. Apparently, there is a sharp enhancement in the resistance of the sample at around the tenth etching step, corresponding to a thickness of about 20-50 nm. At this stage, we looked at another sample of the same batch under AFM. The film was $40 \pm 20 \mathrm{~nm}$ thick. This suggests an average removal of about $7 \mathrm{~nm}$ per etching step. Subsequent to the 12th etching step (not shown in the figure), the sample resistance increased to $\sim 100$ $\mathrm{k} \Omega$. This might point to the widening of the semiconductor energy gap already opened at around the tenth step. On the other hand, according to the theoretical models 2, 13], the semimetal-to-semiconductor (SM-SC) transition thickness should equal the periodicity of the 


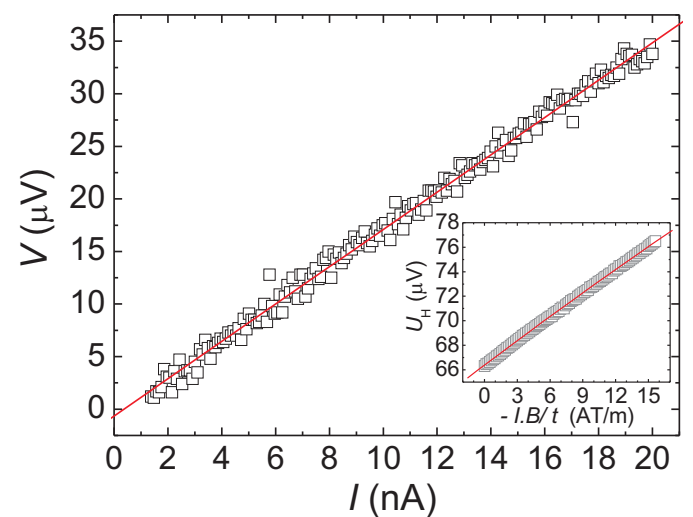

FIG. 5: (Color online) $I V$ characteristic of a solitary bismuth wire with $w \approx 300 \mathrm{~nm}, t \approx 150 \mathrm{~nm}$, and $l=14 \mu \mathrm{m}$ at $T \approx 4.2 \mathrm{~K}$. Note the linearity. (Inset) Dependence of the Hall voltage $U_{\mathrm{H}}$ on the magnetic flux density $B$. Here $I$ is the current passing through the macroscopic Hall slab and $t$ is the thickness. Solid red line is a linear fit. The slope of the line corresponds to $-1 / n q$ with $n$ and $q$ being the volume density and the charge of the carriers, respectively.

oscillations, i.e. $\sim 14 \mathrm{~nm}$ here. A closer look at this issue would be in order. At low temperatures, the expression for the electrical conductivity in the SC regime [13] can be estimated as

$$
R_{0} w_{0} t_{0} \approx R w t \exp \left(-E_{\mathrm{g}} / 2 k_{\mathrm{B}} T\right)
$$

where $E_{\mathrm{g}}=E_{\mathrm{g}}(w, t)$ is the energy gap. $R_{0}, w_{0}$, and $t_{0}$ are the electrical resistance, the width and the thickness at the SM-SC transition. $R$ and $t$ correspond to any arbitrary points on the resistance-thickness plot, subsequent to the transition to the $\mathrm{SC}$ regime. Assuming $w \approx w_{0}$ and substituting the measured values of parameters [Fig. $6(\mathrm{a})]$ in the equation above, $R_{0} \approx 13 \mathrm{k} \Omega, t_{0} \approx 30 \mathrm{~nm}$, $R \approx 22 \mathrm{k} \Omega$ and $t \approx 23 \mathrm{~nm}$, one obtains $E_{\mathrm{g}} \approx 0.5 k_{\mathrm{B}} T$. This is comparable to what one would anticipate in the vicinity of the SM-SC transition, $E_{\mathrm{g}} \sim k_{\mathrm{B}} T$.

The measured data for another sample with $w=280$ $\mathrm{nm}, t=150 \mathrm{~nm}$, and $l=18 \mu \mathrm{m}$ are presented in Fig. 6(b). This sample was damaged after the 16th etching step prior to its characterization at $T \approx 4.2 \mathrm{~K}$. The sample resistance starts exponential growth at around the 14th etching step, corresponding to a transition thickness of about $t_{0} \approx 38-80 \mathrm{~nm}$. The energy gap can be evaluated as explained above. Now, with $R_{0} \approx 100 \mathrm{k} \Omega$, $R \approx 250 \mathrm{k} \Omega$ (estimated based on the resistance value at $77 \mathrm{~K})$, and $t \approx 22-70 \mathrm{~nm}$, we obtain $E_{\mathrm{g}} \approx(0.7-1.6) k_{\mathrm{B}} T$. The puzzling observation in Fig. 6(b) is the systematic decrease of the oscillation amplitudes at lower temperatures. A possible reason could be the formation of ther-
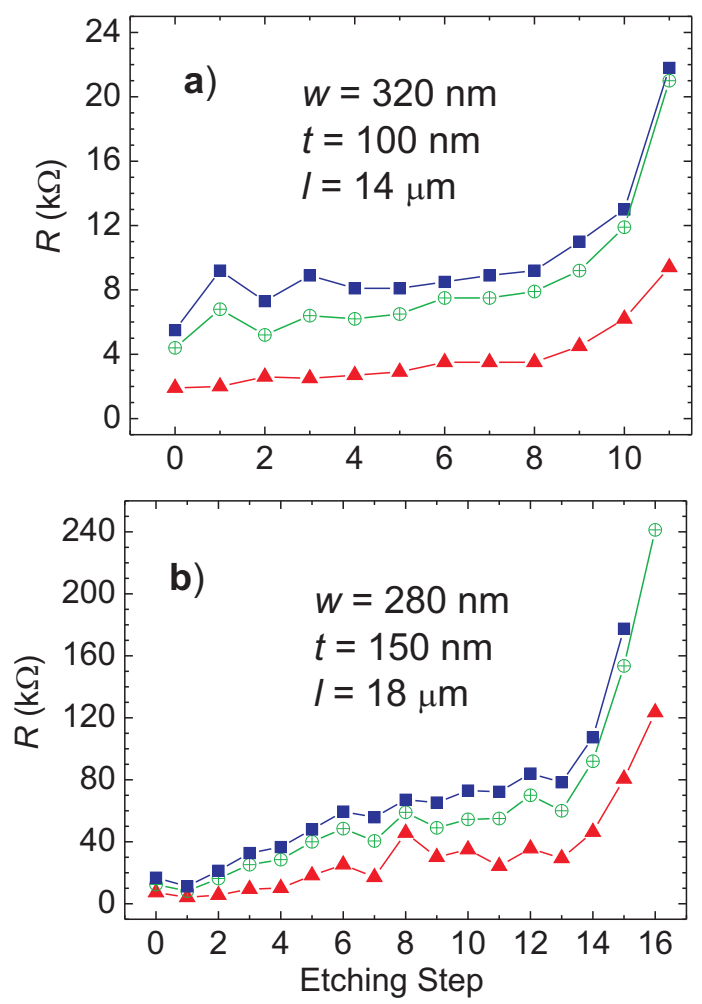

FIG. 6: (Color online) Dependence of the electrical resistance of a bismuth wire on its thickness at liquid helium (solid squares), liquid nitrogen (crossed circles), and room (solid triangles) temperatures. (a) The sample was originally 320 $\mathrm{nm}$ wide, $100 \mathrm{~nm}$ thick, and $14 \mu \mathrm{m}$ long. Each etching step corresponds to a removal of about $5-8 \mathrm{~nm}$ film thickness. (b) Note the unexpected decrease of the oscillation amplitudes at lower temperatures. This sample broke after the 16th etching step, prior to its characterization in liquid helium.

mal stresses within the sample which, in turn, might affect the morphology and the band structure. A thorough explanation, however, is obviously needed. The period of oscillations here, as was the case with the previous sample, is about $10-16 \mathrm{~nm}$. This is not surprising, given the fact that the samples were fabricated under similar conditions. However, the same disagreement between the values obtained from the threshold of the exponential tail and those estimated from the period of the oscillations persists. In addition, in both the samples, in contrast to the theoretical predictions [2, 13], as the SM-SC transition is approached, the oscillations (at a fixed temperature) fade out. These discrepancies may be due to several reasons. Apart from the experimental nonidealities (e.g., the wires are not strictly single crystalline, but consist of an array of crystallites along the wire), in the existing theoretical models, the scattering potential $V_{0}$ as well as the density of states (DOS) were assumed to be ho- 
mogeneous within the wire. In experiments, however, as noted above, the asperity $\eta$ of the sample grows abruptly at reduced dimensions and becomes comparable to the carrier wavelength, $\eta \sim \lambda$. As a consequence, the (grain) boundary scattering plays an increasingly important role and one has $V_{0}=V_{0}(\eta ; w, t)$. Similarly, in a more sophisticated treatment, especially as the sample size shrinks, the DOS should be substituted with the local density of states or with the spectral functions [18]. Furthermore, if the periods of oscillations have to become smaller as the specimens thin (as has been the case in the experimental study of 2D bismuth films [5]), the likeliness of missing the oscillations along the etching steps would increase substantially at lower thicknesses. Also, as verified in the recent experiments [19], the first layers of the grown films may have a different crystal structure. In present study, this would mean alteration of the effective mass values as the film thins. Finally, let us notice that from the observed period of oscillations and the standing wave analogy, one can infer $\lambda \approx 32 \mathrm{~nm}$ for the carrier wavelength and $n \approx 2.5 \times 10^{17} \mathrm{~cm}^{-3}$ for the volume density of the carriers.

To summarize, we have observed QSEs in solitary wires of bismuth. The n-type nanowires were only one singlecrystallite wide. Suppression of the resistance oscillations as the wires thin points to the dominance of surface scattering at reduced dimensions. Our findings could be helpful in further development of scalable solid-state devices comprised of band-engineered semimetals.

We would like to thank A. Schubert for the XRD patterns and K. Arutyunov for his assistance in the Hall measurements.

* Electronic address: shadyar@mpi-halle.de

[1] Yu. F. Ogrin, V. N. Lutskiı̌, and M. I. Elinson, JETP Lett. 3, 71 (1966).

[2] V. B. Sandomirskiı̆, Sov. Phys. JETP 25, 101 (1967).
[3] D. E. Beutler, T. L. Meisenheimer, and N. Giordano, Phys. Rev. Lett. 58, 1240 (1987).

[4] F. Y. Yang, Kai Liu, Kimin Hong, D. H. Reich, P. C. Searson, C. L. Chien, Science 284, 1335 (1999).

[5] E. I. Rogacheva, S. N. Grigorov, O. N. Nashchekina, S. Lyubchenko, and M. S. Dresselhaus, Appl. Phys. Lett. 82, 2628 (2003).

[6] Zhibo Zhang, Xiangzhong Sun, M. S. Dresselhaus, Jackie Y. Ying, and Joseph P. Heremans, Appl. Phys. Lett. 73, 1589 (1998).

[7] Kai Liu, C. L. Chien, and P. C. Searson, Phys. Rev. B 58, R14681 (1998).

[8] J. Heremans, C. M. Thrush, Yu-Ming Lin, S. Cronin, Z. Zhang, M. S. Dresselhaus, and J. F. Mansfield, Phys. Rev. B 61, 2921 (2000).

[9] S. B. Cronin, Ph.D. thesis, Electronic properties of Bi nanowires, Massachusetts Institute of Technology (2002).

[10] P. Chiu and I. Shih, Nanotechnology 15, 1489 (2004).

[11] Y. Tian, G. Meng, S. K. Biswas, P. M. Ajayan, S. Sun, and L. Zhang, Appl. Phys. Lett. 85, 967 (2004).

[12] T. W. Cornelius, M. E. Toimil-Molares, R. Neumann, and S. Karim, J. Appl. Phys. 100, 114307 (2006).

[13] Shadyar Farhangfar, Phys. Rev. B 74, 205318 (2006).

[14] Yu. F. Komnik and E. J. Bukhstab, JETP Lett. 6, 58 (1967).

[15] It is to be noted that the TEM analysis would give information on only a few monolayers of the grown film over a limited surface area of the specimen. The XRD technique instead asks for a much larger film surface (to gain measurable signal) and consequently cannot be used for characterization of a solitary nanostructure. Thus, calling the wires "single crystalline" on the basis of diffraction patterns, as is a common usage in the literature, may not always be unambiguous.

[16] R. T. Isaacson and G. A. Williams, Phys. Rev. 185, 682 (1969).

[17] See, e.g., J. M. Ziman, Electrons and Phonons (Oxford University Press, Oxford, 2001).

[18] See, e.g., John H. Davies, The Physics of LowDimensional Semiconductors (Cambridge University Press, Cambridge, U.K., 1998).

[19] A. Crottini, D. Cvetko, L. Floreano, R. Gotter, A. Morgante, and F. Tommasini, Phys. Rev. Lett. 79, 1527 (1997). 